\title{
Recent progress in nuclear data on secondary particle production and transport by high-energy light/heavy ions
}

\author{
T. Nakamura \\ Cyclotron and Radioisotope Center, Tohoku University, Aoba, Aramaki, Aoba-ku, Sendai 980-8578, Japan
}

\begin{abstract}
The experimental results on DDX (double differential neutron production cross section data), TTY (thick target neutron production yield data), heavy-ion produced neutron transport data, and spallation products production cross section data taken at Heavy Ion Medical Accelerator (HIMAC), National Institute of Radiological Sciences (NIRS), Japan, are presented for heavy ions of He to Xe in the energy range of $100 \mathrm{MeV} /$ nucleon to $800 \mathrm{MeV} / \mathrm{nucleon}$. In addition to the HIMAC results, the following experimental results are presented: 1) neutron yields and shielding data taken recently at heavy ion accelerator of Gesellschaft fuer Schwerionenforschung (GSI), Germany; 2) the neutron production data in the forward direction by 200 to $400 \mathrm{MeV}$ protons measured at cyclotron facility, Research Center for Nuclear Physics (RCNP), Osaka University; 3) some deep penetration shielding experiments using high energy proton accelerators. These experimental results are compared with several Monte Carlo calculations.
\end{abstract}

\section{Introduction}

High-energy heavy-ion accelerator facilities and radioactive beam facilities using the spallation products from intense heavy ion beams are now operating or planned for construction in the world for the discovery of new super-heavy nuclei and unstable nuclei, clinical studies and treatments of the cancer therapy. The high-energy heavy-ion constituents of cosmic radiation must also be considered in space exploration. In these high-energy heavy-particle facilities many secondary particles are created from nucleus-nucleus interactions, and these particles, especially neutrons, can produce radioactivities induced in accelerator and structural materials, air, water, and soil, and can penetrate through the facility building into the surrounding environment. It is therefore quite necessary to evaluate the emission of secondary particles, especially neutrons and the creation of the residual nuclei in various materials, in order to estimate the source terms of accelerator shielding design, and also to calculate the dose delivered in the human body during the therapeutic irradiation.

In this paper, the experimental results on DDX (double differential neutron production cross section data), TTY (thick target neutron production yield data), heavy-ion produced neutron transport data, and spallation products production cross section data taken at Heavy Ion Medical Accelerator (HIMAC), National Institute of Radiological Sciences (NIRS), Japan, are presented for heavy ions of He to Xe in the energy range of $100 \mathrm{MeV} /$ nucleon to $800 \mathrm{MeV} /$ nucleon. In addition to the HIMAC results, the following experimental results are presented: 1) neutron yields and shielding data taken recently at heavy ion accelerator of Gesellschaft fuer Schwerionenforschung (GSI), Germany; 2) the neutron production data in the forward direction by 200 to $400 \mathrm{MeV}$ protons measured at cyclotron facility, Research Center for Nuclear Physics (RCNP), Osaka University; 3) some deep penetration shielding experiments using high energy proton accelerators.

Comprehensive experimental results at HIMAC with some experimental results taken at other heavy-ion accelerators are summarized in a handbook [1], with the moving-source parameterization of thick-target neutron yields and neutronproduction cross sections, and application of data to benchmark transport model calculations. These experimental results will be quite useful as benchmark data for Monte Carlo code analysis and evaluation.

\section{Secondary neutron yields from thick targets}

As one example of comprehensive experimental results summarized in the handbook [1], figure 1 gives the neutron energy spectra at angles of 0 to 90 degrees produced from thick (stopping length) $\mathrm{C}$ and $\mathrm{Cu}$ targets bombarded by $400 \mathrm{MeV} /$ nucleon Fe ions at HIMAC [2]. The neutron spectra were obtained by the TOF method using NE213 scintillators with NE102A plastic veto detectors. The measured spectra are compared with the PHITS (Particle and Heavy Ion Transport System) Monte Carlo code [3]. Three components can be clearly seen in the spectra. In general, the spectra in the forward direction have a broad peak at the high-energy end. The peak energy usually occurs at about 60 to $70 \%$ of the beam energy per nucleon. As the target mass becomes lighter and the projectile mass increases, the high-energy peak becomes more prominent. Most of the neutrons in this high-energy, forward region come from the breakup of the projectile and direct knock-on processes. Neutrons with energies up to 2.5 times the incoming beam energy per nucleon can be produced by these processes. At energies below $20 \mathrm{MeV}$, the spectra are dominated by the breakup of the target. Because the target remnant is moving slowly in the lab. frame, that source of neutrons is essentially isotropic. As such, target-like neutrons can be seen at all angles. As target mass increases, the relative contribution to the overall spectra from target breakup increases. At intermediate energies and intermediate angles, the spectra are dominated by the decay of the overlap region, where a sizeable number of projectile nucleons and target nucleons mix and can undergo several nucleon-nucleon 

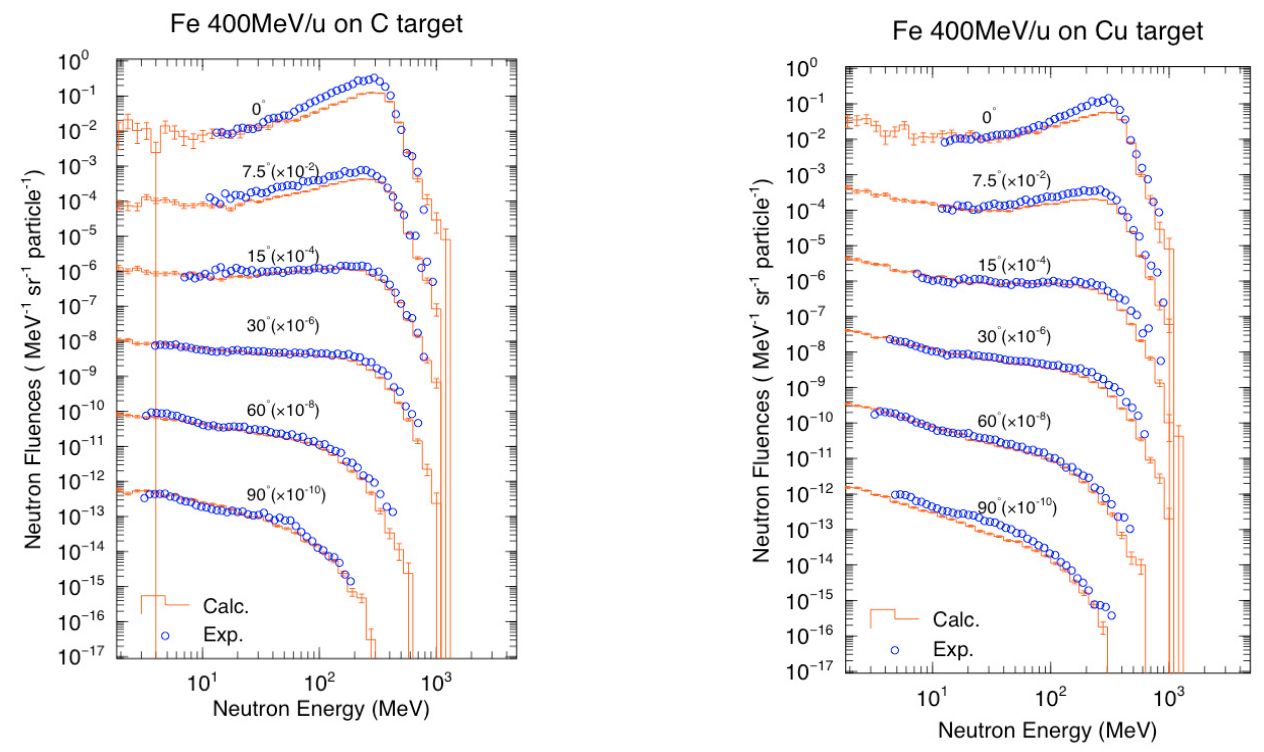

Fig. 1. Comparison of measured neutron spectra at 0 to 90 degrees from thick (stopping length) $\mathrm{C}$ and $\mathrm{Cu}$ targets bombarded by $400 \mathrm{MeV} /$ nucleon Fe ion with PHITS calculations [2,3].

collisions. The PHITS calculation gives good agreement with the measured spectra, excluding the high-energy region at 0 degree.

The TTY experiment was performed at GSI using a ${ }^{238} \mathrm{U}$ beam of $1 \mathrm{GeV} /$ nucleon energy impinging on a 20 -cm thick Fe target. Neutrons were detected in the Large Area Neutron Detector (LAND) with an active area of $2 \mathrm{~m} \times 2 \mathrm{~m}$ in neutron angle of 0 to 20 degrees and in energy above $50 \mathrm{MeV}$ as shown in figure 2 [4]. The measured spectra are compared with two Monte Carlo codes, PHITS [3] and FLUKA [5]. Two codes give underestimation, especially at 0 degree, to the measured spectra in higher energy region, as also seen in figure 1 .

The neutron spectra generated at 0 degree from thick (stopping length) targets of carbon, aluminum, iron and lead, bombarded by $250,350 \mathrm{MeV}$ protons were also measured in the ring cyclotron facility at Research Center for Nuclear Physics (RCNP), Osaka University, Japan with the TOF method using an NE213 scintillator. Figure 3 exemplifies the neutron energy spectra at 0 degree for carbon and iron targets compared with the PHITS and MCNPX [6] calculations for $350 \mathrm{MeV}$ proton bombardment [7]. Calculated results of PHITS with Bertini/GEM and MCNPX with LA150+Bertini underestimate the experimental results in the neutron energy range from $30 \mathrm{MeV}$ to incident energy, as well as the underestimation of the calculations found in the thick iron target experiment at 0 degree for $210 \mathrm{MeV}$ proton at Institute of Physical and Chemical Research (RIKEN) [8]. Those may result from the underestimation of neutron-production DDX (Double Differential Cross Sections) at small angles and the strong self-shielding in target nucleus. On the other hand, the calculated result with the JENDL-HE file [9] gives good agreement with experimental results below $100 \mathrm{MeV}$ on graphite and below $150 \mathrm{MeV}$ on aluminum and iron.

The neutron spectra generated at 0 degree from thin and thick targets are very scarce and all calculations give largely underestimated results, so it is strongly required to get more experimental results and also to improve the calculation models and nuclear data in the forward direction.

\section{Secondary neutron yields from thin targets}

As one example of comprehensive experimental results summarized in the handbook [1], figure 4 gives the neutron energy spectra at angles of 5 to 80 degrees produced from thin $\mathrm{Cu}$ target bombarded by $400 \mathrm{MeV} /$ nucleon $\mathrm{Ne}$ and $\mathrm{Ar}$ ions at HIMAC $[10,11]$. The neutron spectra were also obtained by the TOF method using NE213 scintillators. The measured

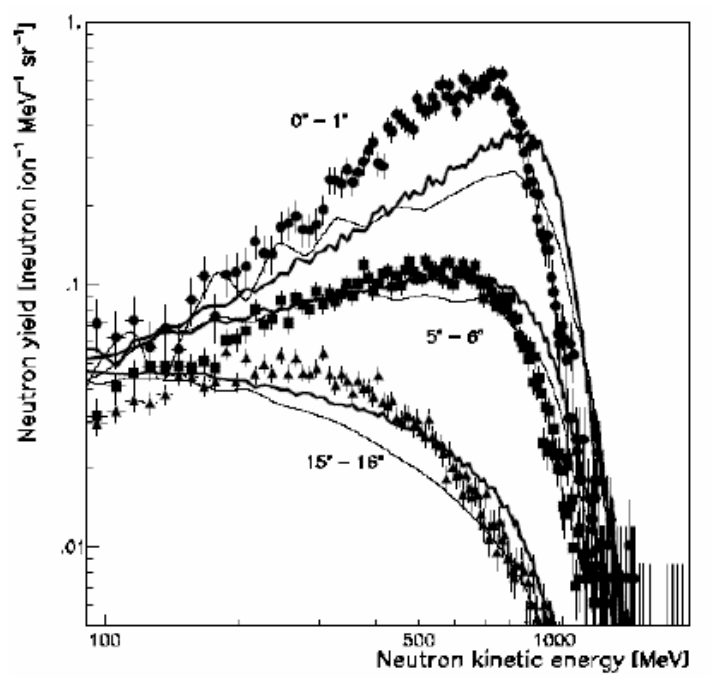

Fig. 2. Neutron yields vs. neutron kinetic energy observed for a beam of $238 \mathrm{U}$ ( $1 \mathrm{GeV} /$ nucleon) on a thick Fe target at polar angles as indicated. Symbols denote the experimental data; thick (thin) lines represent results obtained with the FLUKA (PHITS) code [4]. 

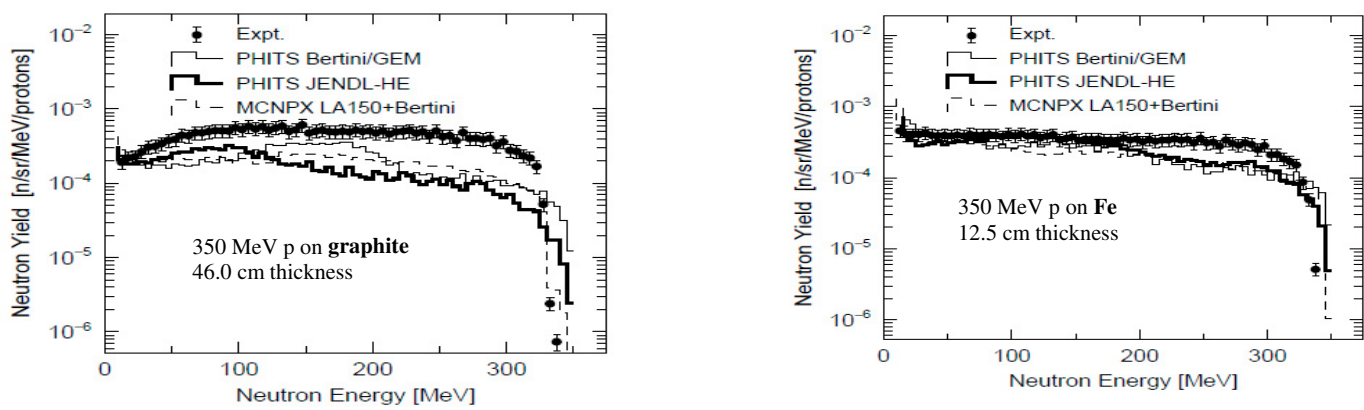

Fig. 3. Comparison of measured neutron spectra at 0 degree from thick graphite and iron targets bombarded by $350 \mathrm{MeV}$ protons with PHITS and MCNPX calculations [7].
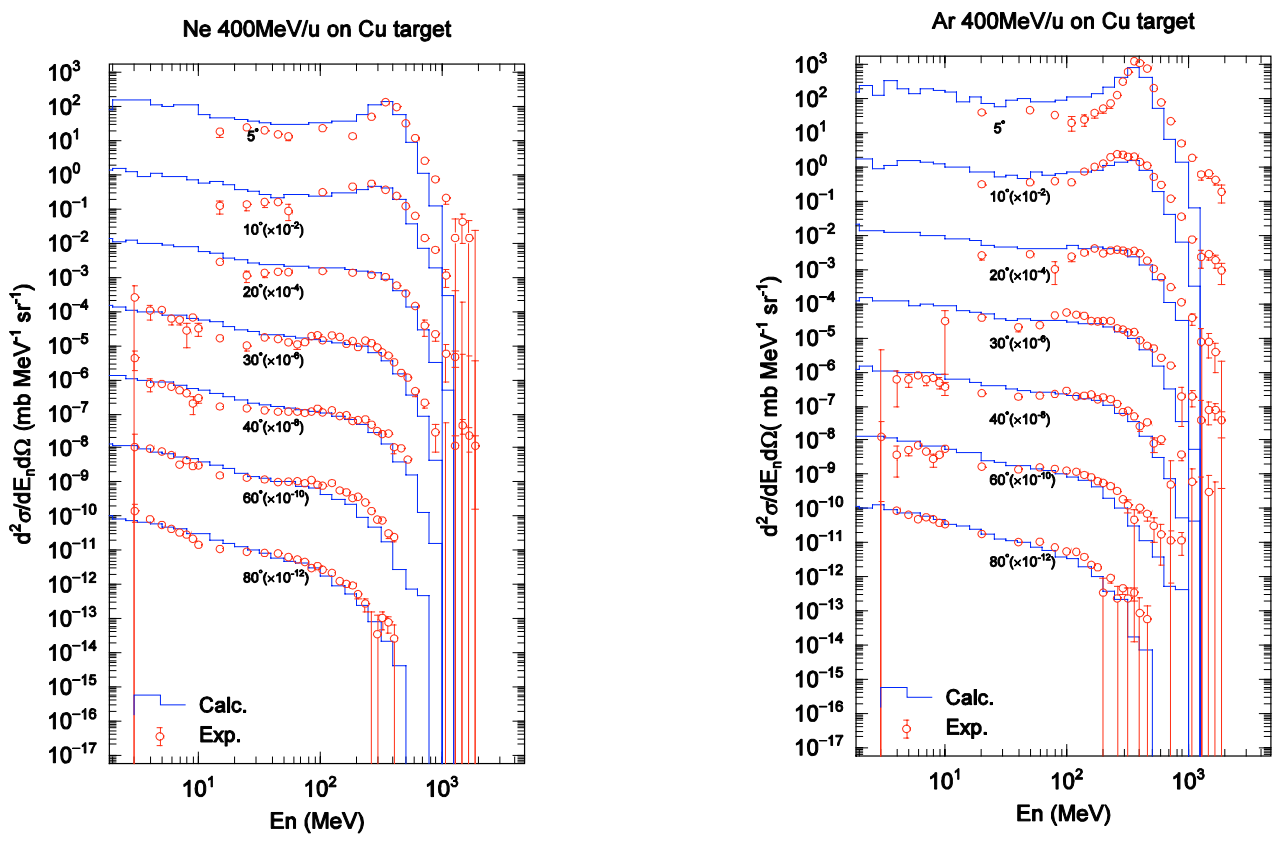

Fig. 4. Comparison of measured neutron spectra at 5 to 80 degrees from thin $\mathrm{Cu}$ target bombarded by $400 \mathrm{MeV} / \mathrm{nucleon} \mathrm{Ne}$ and Ar ions with PHITS calculations $[10,11]$.

spectra are compared with the PHITS calculation. At forward angles, there is a prominent peak centered near the beam energy per nucleon. As the angle increases, the prominence of the peak decreases to a point at about $20^{\circ}$ where the peak is insignificant. The high-energy neutrons in the region of this forward peak come mainly from the breakup of the projectile, along with direct knock-out neutrons from the target. Neutrons are detected at energies 2 to 3 times the beam energy per nucleon, which is a phenomenon attributable to the collective Fermi motion inside the nucleus adding a momentum kick during the collision. At energies below $10-20 \mathrm{MeV}$, the spectra are dominated at all angles by the decay of the target remnant. The exponential behavior of the cross section with energy in this region suggests the target remnant decays by an equilibrium process. At intermediate energies (above 10 to $20 \mathrm{MeV}$, below the beam energy per nucleon), there is a component that becomes less pronounced as the angle increases. This component is dominated by the pre-equilibrium decay of the overlap region between the projectile and the target. The PHITS calculation gives reasonably good agreement with the measured results at all angles from 5 to 80 degrees. This means that the disagreement between experiment and simulation is remarkable only around 0 degree.

Figure 5 gives the total cross sections from 0 to 180 degrees deduced by integrating the measured spectra above $10 \mathrm{MeV}$ and adding the angular-integrated data from 0 to 90 degrees, together with the calculated total cross sections from 90 to 180 degrees, which are estimated through the exponential fitting of angular distribution data from 0 to 90 degrees [11]. It is found that the total cross sections can be estimated using a simple parameterization that is based on the geometric cross section and the number of projectile and target neutrons from each system.

\section{Measurements of high energy neutrons behind shielding}

Recently, measurements have been made of neutrons behind shielding materials at heavy-ion accelerator facilities [1]. Experiments done with $400-\mathrm{MeV} /$ nucleon $\mathrm{C}$ beams were performed at HIMAC [12] using the self-TOF detector 


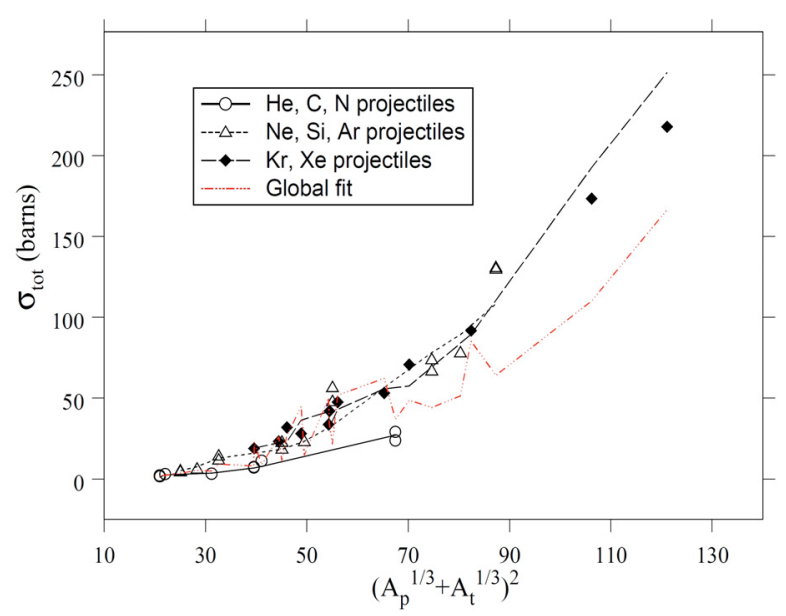

Fig. 5. Total neutron production cross sections above $10 \mathrm{MeV}$ and angles between 0 and 180 degrees as a function of a parameter that is proportional to the geometric cross sections [11].
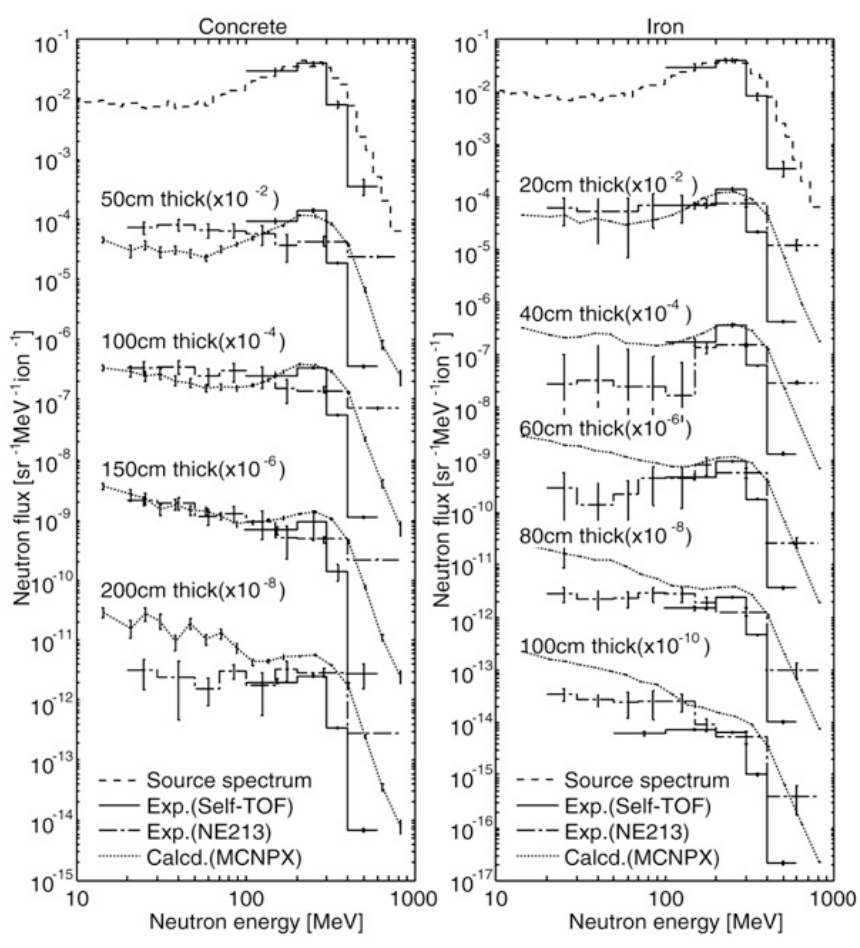

Fig. 6. Comparison of measured and calculated neutron energy spectra penetrating through (a) concrete and (b) iron shields. Solid lines are the measured results of the self-TOF detector, dot-dash lines are of the NE213 detector. Dotted lines are the MCNPX calculations and the broken line is the source neutron spectrum generated from a $\mathrm{Cu}$ target bombarded by $400 \mathrm{MeV} /$ nucleon $\mathrm{C}$ ions [12].

and the NE213 scintillator. The shielding experiments of concrete and iron were done at the NSCL (National Superconducting Cyclotron Laboratory), Michigan State University using a heavy metal (Hevimet) target bombarded by $155 \mathrm{MeV} /$ nucleon $\mathrm{He}, \mathrm{C}$ and $\mathrm{O}$ ions [13]. The neutron spectra were measured at several positions inside and outside the irradiation room at GSI [14]. The latter two measurements were done with the multi-sphere spectrometer, Bonner ball.
In the HIMAC series of experiments, neutrons were produced by stopping $400 \mathrm{MeV} /$ nucleon ${ }^{12} \mathrm{C}$ ions in a $5-\mathrm{cm}$ thick $\mathrm{Cu}(10 \times 10 \mathrm{~cm}$ square $)$ target. The concrete and iron shields were $50-$ and $10-\mathrm{cm}$ thick slabs, respectively, both $100 \times 100 \mathrm{~cm}$ square, and they were placed centered and normal to the beam axis. Figure 6 shows the neutron fluxes $\left(\mathrm{str}^{-1} \cdot \mathrm{MeV}^{-1}\right.$. ion $\left.^{-1}\right)$ behind $0,50,100,150,200 \mathrm{~cm}$ of concrete, and $0,20,40,60,80,100 \mathrm{~cm}$ of iron. The self-TOF detector gives the neutron spectra above the low-energy threshold of $100 \mathrm{MeV}$ (the threshold is due to low energy recoils that range out in the radiator), and below $600 \mathrm{MeV}$ (due to a lack of statistically-significant events). The spectra have a broad peak around 200 to $300 \mathrm{MeV}$. Little softening of the spectra can be seen with increasing shield thickness. The NE213 detector gives the neutron spectra from 20 to $800 \mathrm{MeV}$. The spectra given both by the self-TOF detector and the NE213 detector are generally in good agreement with each other, although the broad peaks are not seen in the NE213 spectra. The dashed lines show MCNPX calculations of the spectra. The calculations, in general, give a harder spectrum than do the measurements. Below $100 \mathrm{MeV}$, the calculations overestimate the data as the shielding thickness increases. Between 100 and $400 \mathrm{MeV}$, the agreement between experiment and calculation is good. The agreement between calculation and experiment is quite good over the entire energy range (20-800 MeV) at $20-\mathrm{cm}$ of iron shielding, and at 50-, 100-, and 150-cm of concrete shielding.

A series of high-energy neutron experiments for accelerator shielding research has been performed at the neutron time-of-flight (TOF) beam course at RCNP [15]. In this study, a quasi-monoenergetic neutron field was developed using the ${ }^{7} \mathrm{Li}(\mathrm{p}, \mathrm{n})^{7} \mathrm{Be}$ reaction in the energy range from 250 to $390 \mathrm{MeV}$. Proton beams of 250, 350 and $392 \mathrm{MeV}$ energies extracted from the cyclotron were transported to the experimental hall and impinged onto a $10 \mathrm{~mm}$ thick Li target placed in a vacuum chamber. Protons passing through the target were swept out towards the beam dump by the swinger magnet to measure the proton beam intensity with a Faraday cup. Neutrons produced at 0 degree from the Li target were extracted into the TOF room of $100 \mathrm{~m}$ length through a concrete collimator of $10 \mathrm{~cm} \times$ $12 \mathrm{~cm}$ aperture and $150 \mathrm{~cm}$ thickness, while charged particles were rejected by a vertical bending magnet located in the collimator. The shielding experiment was performed with 250 and $350 \mathrm{MeV}$ p-Li quasi-monoenergetic neutrons. Concrete and iron shields of $120 \mathrm{~cm} \times 120 \mathrm{~cm}$ sizes having $10 \mathrm{~cm}$ and $5 \mathrm{~cm}$ thickness, respectively, were added to change the total thickness up to $2 \mathrm{~m}$ for concrete and $1 \mathrm{~m}$ for iron. They were fixed just after the concrete collimator. The NE213 scintillator and the multi-moderator detector with ${ }^{3} \mathrm{He}$ counter, Bonner ball, were set behind the shield to get the neutron energy spectra down to thermal energy. Figure 7 gives the preliminary results of energy spectra penetrated through iron and concrete shields of several thicknesses measured with the Bonner ball [16]. A sharp monoenergetic peak can clearly be seen at $248 \mathrm{MeV}$ coming from a $250 \mathrm{MeV}$ p-Li source neutron. The spectra become softer having larger low-energy components with shield thickness and the spectra behind iron indicate big broad bump in the energy region around several hundreds keV.

For higher energy proton accelerators, Nakao et al. performed the shielding experiment using a $120 \mathrm{GeV} / \mathrm{c}$ 

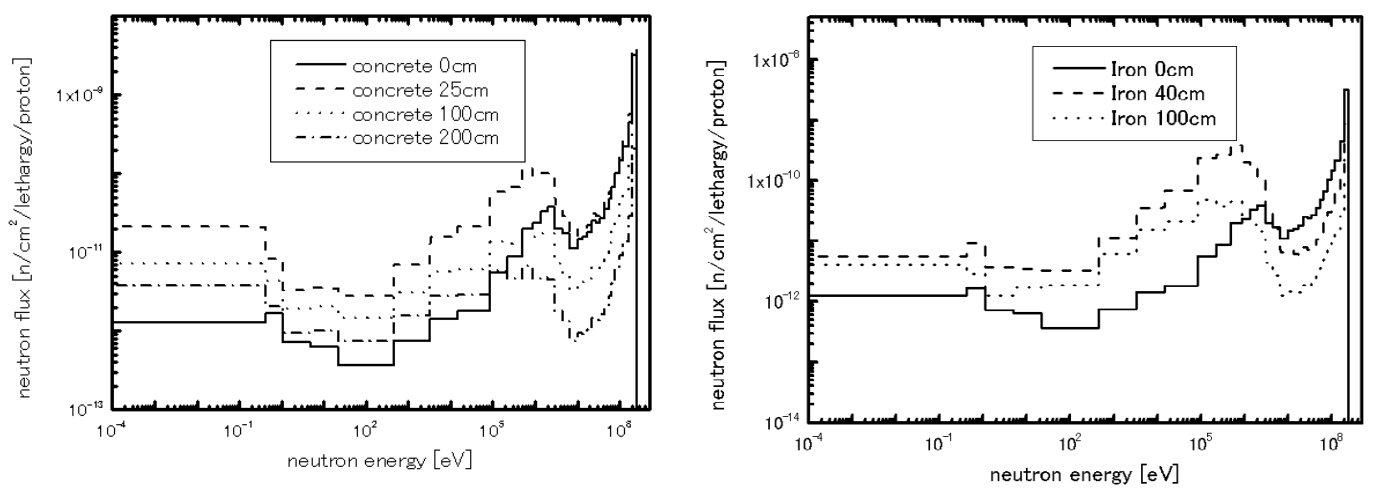

Fig. 7. Measured neutron spectra penetrated through concrete and iron shields of different thickness with the source neutron spectrum of quasi-monoenergetic $248 \mathrm{MeV}$ energy [16]. The data were taken with the Bonner ball.
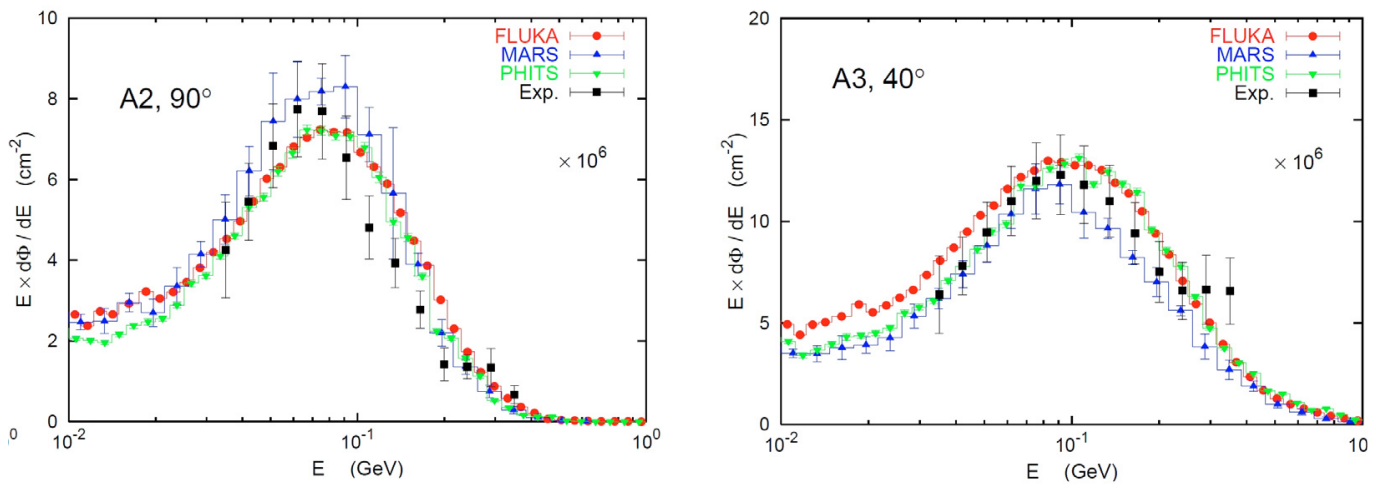

Fig. 8. Comparison of measured neutron spectra at 40 and 90 degrees to the beam direction of $120 \mathrm{GeV} / \mathrm{c}$ positive hadrons (protons and pions) behind the 80-cm thick concrete shield with FLUKA, PHITS and MARS calculations [17].

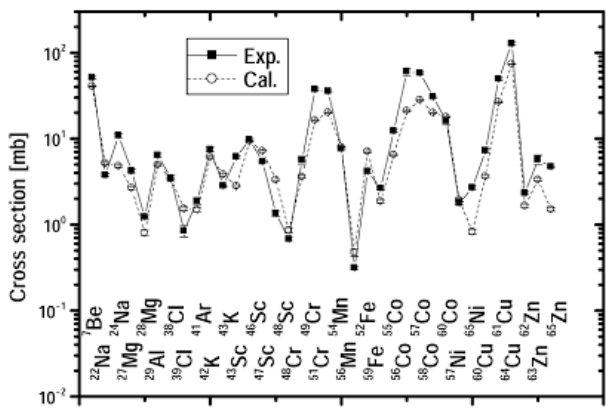

(c) $400 \mathrm{MeV} /$ nucleon $\mathrm{Ne}$

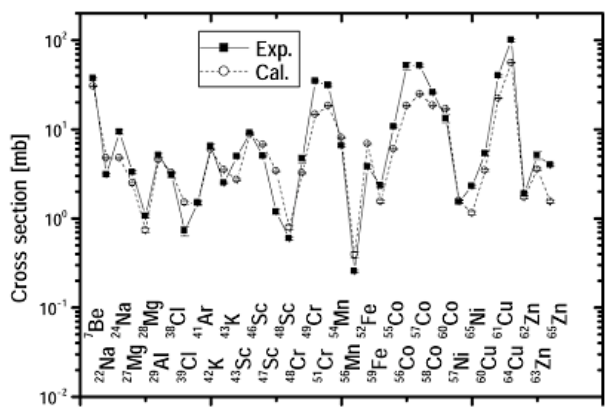

(d) $400 \mathrm{MeV} /$ nucleon $\mathrm{C}$

Fig. 9. Comparison of measured and calculated reaction cross sections of spallation products in $\mathrm{Cu}$ for $400 \mathrm{MeV} /$ nucleon $\mathrm{C}$ and Ne projectiles with PHITS calculations [20].

positive hadron beam (mainly a mixture of $34.8 \%$ protons, $60.7 \%$ pions, and $4.5 \%$ kaons) facility at CERF (CERN-EU High Energy Reference Field), CERN [17]. At CERF, the beam was injected on a cylindrical $\mathrm{Cu}$ target of 7-cm diameter by $50-\mathrm{cm}$ length. The neutron energy spectra produced from the target were measured at various longitudinal locations behind shields of $80-$ and $160-\mathrm{cm}$ thick concrete and $40-\mathrm{cm}$ thick iron using the NE213 scintillator coupled with the NE102A veto counters. Figure 8 exemplifies the measured neutron spectra from 12 to $380 \mathrm{MeV}$ at 40 and 90 degrees to the beam direction behind the $80-\mathrm{cm}$ thick concrete shield.
The measured spectra having a broad peak around $100 \mathrm{MeV}$ are compared with three Monte Carlo calculations by MARS [18], FLUKA [5] and PHITS [3]. The agreement between experiment and calculation is pretty good.

\section{Production cross sections of spallation products created in heavy ion reactions}

To this end, the production cross sections for various spallation products have been measured from heavy-ion 
reactions by several groups [1], additionally for $\mathrm{Cu}$ target after $800 \mathrm{MeV} /$ nucleon Ar irradiation at GSI [19].

The HIMAC series of experiments [20] used a target stack comprised of two to seven 5 -mm thick $\mathrm{Cu}$ plates made of $10 \mathrm{~cm}$ by $10 \mathrm{~cm}$ squares. In between each plate, samples of $\mathrm{C}(0.2-\mathrm{mm}$ thick, $5 \times 5 \mathrm{~cm}$ square $), \mathrm{Al}(0.1-\mathrm{mm}$ thick, $9 \times$ $\left.10 \mathrm{~cm}^{2}\right)$, and $\mathrm{Cu}\left(0.1-\mathrm{mm}\right.$ thick, $\left.9 \times 10 \mathrm{~cm}^{2}\right)$ were placed in order to measure the spatial distributions of spallation products, and to determine the energy dependence on the cross section. Foils of the same thickness were placed at the front of the stack to measure the reaction cross sections and mass-yield distributions. The data can be summed over the same mass numbers to produce mass-yield distributions. As one example, figure 9 shows the mass-yield distributions (in $\mathrm{mb}$ ) of 35 nuclides from ${ }^{7} \mathrm{Be}$ to ${ }^{65} \mathrm{Zn}$ for the $400-\mathrm{MeV} /$ nucleon $\mathrm{C}$ and $\mathrm{Ne}$ ions interacting in a $\mathrm{Cu}$ target compared with PHITS calculations. The PHITS calculations give good agreement with the experimental results, excluding an underestimation for some nuclides.

\section{Conclusion}

The work and data described here result from the efforts of scientists from Japan, the US, and Europe during the past 25 years. We have focused on research that is applicable to the general field of high-energy ( $>100 \mathrm{MeV} /$ nucleon) light/heavyion transport. More specific applications in that field include accelerator design and shielding, medical physics and heavyion radiotherapy, and space-radiation transport and shielding design. It was our pleasure to survey the field to collect the most comprehensive data set we could find in four main areas: thick-target neutron yield measurements, neutron production cross sections, measurements of neutrons behind shielding, and spallation products production cross sections.

The author wishes to thank L. Heilbronn, H. Iwase and K. Kosako for giving him the graphs used in this paper.

\section{References}

1. T. Nakamura, L. Heilbronn, Handbook on Secondary Particle Production and Transport by High-Energy Heavy Ions (World Scientific, Singapore, 2006).

2. T. Kurosawa, N. Nakao, T. Nakamura et al., Phys. Rev. C 62, 044615 (2000).

3. H. Iwase, K. Niita, T. Nakamura, J. Nucl. Sci. Technol. 39, 1142 (2002).

4. O. Yordanov, K. Gunzert-Marx et al., Nucl. Instrum. Meth. B 240, 863 (2005).

5. A. Fasso, A Ferrari, J. Ranft, P.R. Sala, in Proceedings of the Monte Carlo 2000 Conference, Lisbon, 2000 (Springer-Verlag, Berlin, 2001), p. 955.

6. L.S. Water (ed.), Los Alamos National Laboratory, LA-CP-02408, 2002.

7. Y. Iwamoto, S. Taniguchi, N. Nakao et al., Nucl. Instrum. Meth. A 562, 789 (2006).

8. S. Yonai, T. Kurosawa, H. Iwase et al., Nucl. Instrum. Meth. A 515, 733 (2003).

9. Y. Watanabe et al., AIP Conf. Proc. 769, 1646 (2005).

10. Y. Iwata, T. Murakami, H. Sato et al., Phys. Rev C 64, 054609 (2001) .

11. L. Heibronn, C.J. Zeitlin, Y. Iwata et al., Nucl. Sci. Eng. (in press).

12. M. Sasaki, E. Kim, T. Nunomiya et al., Nucl. Sci. Eng. 141, 140 (2003).

13. G.I. Britvich, A.A. Chumakov, R.M. Ronningen et al., Rev. Sci. Instrum. 70, 2314 (1999).

14. H. Iwase, B. Wiegel, G. Fehrenbacher et al., Radiat. Protec. Dosim. 116, 640 (2005).

15. S. Taniguchi, N. Nakao, T. Nakamura et al., Radiat. Protec. Dosim. (in press).

16. H. Yashima (2006) (private communication).

17. N. Nakao, S. Taniguchi, S. Rokni et al., Nucl. Instrum. Meth. A 562, 950 (2006).

18. N.V. Mokhov, Fermilab-FN-628, 1995.

19. A. Fertman, A. Golubev, B. Sharkov et al. (2005) (private communication).

20. H. Yashima, Y. Uwamino, H. Iwase et al., Radio Chimica Acta 91, 689 (2003); ibid., Nucl. Instrum. Meth. B 226, 243 (2004). 